\title{
A MODEL FOR GENERAL PERIODIC EXCITATION WITH RANDOM DISTURBANCE AND ITS APPLICATION
}

\author{
Y. S. Zhou, Z. K. Hou, ${ }^{*}$ M. F. Dimentberg and M. N. Noori \\ Department of Mechanical Engineering, Worcester Polytechnic Institute, Worcester, \\ MA 01609, U.S.A.
}

\begin{abstract}
Many vibration problems involve a general periodic excitation such as those of a triangular or rectangular waveform. In practice, the periodic excitation may become disordered due to uncertainties. This paper presents a stochastic model for general periodic excitations with random disturbance, which is constructed by introducing random amplitude and phase disturbances to individual terms in the Fourier series of the corresponding deterministic periodic function. Mean square convergence of the random Fourier series are discussed. Monte Carlo simulation of disordered sawtooth, triangular, and quadratic wave forms are illustrated. An application of the excitation is demonstrated by vibration analysis of a single-degree-of-freedom (SDOF) hydraulic valve system subjected to a disordered periodic fluid pressure. In the present study only the phase disturbance is considered. Effects of the intensity of phase modulation on up to fourth order moment response and the convergence rate of the random Fourier series are studied by numerical results. It is found that a small random disturbance in a general periodic excitation may significantly change the response moment.
\end{abstract}

\section{INTRODUCTION}

In many engineering problems, sources of vibration are considered to be periodic. In practice, however, the excitation may not always be perfectly periodic due to uncertainties, and disorders in both amplitude and phase may be observed. It is very important to take into account the random deviations from perfect periodicity. This paper presents a stochastic model for general periodic excitation with random disturbance, which is constructed by introducing random amplitude and phase disturbances into individual terms in the Fourier series of the corresponding deterministic periodic function. In the present study, the discussion is limited to the case of phase modulation alone and these phase modulations in different terms are independent zero-mean white noise processes.

The proposed model is a further extension of the model of single harmonic excitation with correlated random amplitude and phase disturbances, which was established by Hou et al. [1,2]. An earlier version for phase modulation alone was originally used by Dimentberg [3] and Wedig [4] in the investigation of Mathieu type stochastic system and it was later applied by Dimentberg to the studies of parametric excitation of a straight pipe due to slug flow of a two-phase fluid [5], and stability and subcritical dynamics of structures with spatially disordered traveling parametric excitation [6]. Lin and Li also used

* Correspondent. 
the model in the stability analysis of bridges in turbulent flow [7]. The non-Gaussian nature of stationary response of SDOF systems under harmonic excitation with random phase modulation has been analyzed quantitatively by Dimentberg et al. [8]. The excess factor was calculated and the normalization effect with increasing excitation/ system bandwidth ratio had been demonstrated. Hou et al. [9] later developed a non-stationary version of the model and studied the effects of non-stationarity including build-up, decay, and duration of the phase modulation on the system response. As with its ancestors, the proposed model is inherently non-Gaussian in nature. Furthermore, the potential applications of the model seem to be much broader than just those describing imperfectly periodic phenomena. Indeed, the model is quite versatile in describing spectral properties of random processes. Since the model is a strongly non-Gaussian process, it can be easily incorporated into structural dynamic analysis by stochastic differential calculus and method of moments. The potential field of applications may include offshore mechanics, with typical high-amplitude nonlinear ocean waves of nonsinusoidal pattern.

In this study, the mean-square convergence of the random Fourier series is discussed. Numerical simulation of the model are illustrated for wave forms with different degrees of continuity including the saw, triangle, and quadratic waves. The effects of random phase modulations can be clearly observed. Application of the model is demonstrated by vibration analysis of a hydraulic control system subjected to a fluid pressure. The valve is modelled as a linear SDOF mass-spring-dashpot system and the fluid pressure is represented by a randomly disordered periodic triangular waveform. Response of the system is analyzed by the method of moments, and response moments up to fourth order are obtained numerically. The effects of uncertainties in the periodic excitation on moment response and truncation error of the random Fourier series are discussed.

\section{A MODEL FOR RANDOM GENERAL PERIODIC PROCESSES AND ITS CONVERGENCE}

Let $Y(t)$ be a basically periodic process but with small random deviation from perfect periodicity. By adding random phase modulation to each term in equation (1), $Y(t)$ can be modelled as a random Fourier series:

$$
Y(t)=\frac{a_{0}}{2}+\sum_{i=1}^{\infty} a_{i} \cos \theta_{i}(t)+\sum_{i=1}^{\infty} b_{i} \sin \theta_{i}(t), \quad \frac{\mathrm{d} \theta_{i}(t)}{\mathrm{d} t}=\mathrm{i} \omega_{0}+\xi_{i}(t),
$$

where $a_{0}, a_{i}, b_{i}(i=1,2, \ldots)$ are deterministic constants which are in fact Fourier coefficients of the corresponding undisordered periodic function, $\omega_{0}=2 \pi / T$ in which $T$ is the periodic of the corresponding undisturbed periodic function, $\xi_{i}(t)$ is a stationary white noise random process with zero-mean and intensity $D_{i}$, i.e.,

$$
\mathrm{E}\left[\xi_{i}(t)\right]=0, \quad \mathrm{E}\left[\xi_{i}(t) \xi_{i}(t+\tau)\right]=D_{i} \delta(\tau), \quad i=1,2, \ldots
$$

In equation (3), $\delta(\cdot)$ is the Dirac delta function and $E[\cdot]$ is the mathematical expectation operator. In this study, random disturbances $\xi_{i}(t)$ of the $i$ th harmonic term $(i=1,2, \ldots)$ are assumed to be independent of each other which leads to

$$
\mathrm{E}\left[\xi_{i}(t) \xi_{j}(t+\tau)\right]=\mathrm{E}\left[\xi_{j}(t) \xi_{i}(t+\tau)\right]=0, \quad i, j=1,2, \ldots, \quad i \neq j .
$$

The random process $Y(t)$ defined by equations (1) through (4) represents a disordered periodic process. In the case of no random phase disturbances $\left(D_{i}=0, i=1,2, \ldots\right), Y(t)$ reduces to a deterministic general periodic function $F(t)$ with a minimum period of $T$. 
$F(t)$ can be formally expanded into the Fourier series

$$
F(t)=\frac{a_{0}}{2}+\sum_{i=1}^{\infty} a_{i} \cos \mathrm{i} \omega_{0} t+\sum_{i=1}^{\infty} b_{i} \sin \mathrm{i} \omega_{0} t
$$

where

$$
a_{i}=\frac{2}{T} \int_{0}^{T} F(t) \cos \mathrm{i} \omega_{0} t \mathrm{~d} t, \quad i=0,1,2, \ldots ; \quad b_{i}=\frac{2}{T} \int_{0}^{T} F(t) \sin \mathrm{i} \omega_{0} t \mathrm{~d} t, \quad i=1,2, \ldots
$$

The response of linear system to $F(t)$ can then be determined by the principle of superposition. Conditions for convergence of a deterministic Fourier series are referred to Churchill and Brown [10].

To evaluate the probabilistic characteristics of random Fourier series $Y(t)$, a general term of $Y(t)$ is examined. Let

$$
Y_{1 i}(t)=\cos \theta_{i}(t), \quad Y_{2 i}(t)=\sin \theta_{i}(t), \quad i=1,2, \ldots
$$

Both $Y_{1 i}(t)$ and $Y_{2 i}(t)$ are zero-mean randomly disordered harmonic processes [8]. The probability density functions of $Y_{1 i}(t)$ and $Y_{2 i}(t)$ are

$$
p\left(Y_{1 i}\right)=1 /\left(\pi \sqrt{1-Y_{1 i}^{2}}\right), \quad p\left(Y_{2 i}\right)=1 /\left(\pi \sqrt{1-Y_{2 i}^{2}}\right), \quad i=1,2, \ldots
$$

Note that $Y_{1 i}$ and $Y_{2 i}$ are bounded by \pm 1 .

It has been shown that

$$
\mathrm{E}\left[Y_{1 i}^{2}\right]=\mathrm{E}\left[Y_{2 i}^{2}\right]=1 / 2, \quad i=1,2, \ldots
$$

The auto-correlation functions of $Y_{1 i}(t)$ and $Y_{2 i}(t)$ are equal to each other [7].

$$
R_{Y 1 i}(\tau)=R_{Y 2 i}(\tau)=\frac{1}{2} \cos \left(\mathrm{i} \omega_{0} \tau\right) \mathrm{e}^{\left(-D_{i} / 2\right)|\tau|}, \quad \tau=t_{2}-t_{1}, \quad i=1,2, \ldots
$$

Based on equations (8)-(11), the mean value, mean square value, and autocorrelation function of random Fourier series $Y(t)$ can be derived:

$$
\begin{aligned}
& \mathrm{E}[Y(t)]=\frac{a_{0}}{2}+\sum_{i}^{\infty} a_{i} \mathrm{E}\left[Y_{1 i}(t)\right]+\sum_{i}^{\infty} b_{i} \mathrm{E}\left[Y_{2 i}(t)\right]=\frac{a_{0}}{2} \\
& \mathrm{E}\left[Y^{2}(t)\right]=\mathrm{E}\left[\left\{\frac{a_{0}}{2}+\sum_{i=1}^{\infty} a_{i} Y_{1 i}(t)+\sum_{i=1}^{\infty} b_{i} Y_{2 i}(t)\right\}^{2}\right]
\end{aligned}
$$

Since one assumes that the random disturbance $\xi_{i}(t)$ in each harmonic term is independent of the others, the expectation values of all cross-terms should be zero:

$$
\mathrm{E}\left[Y_{1 i}(t) Y_{1 j}(t)\right]=\mathrm{E}\left[Y_{2 i}(t) Y_{2 j}(t)\right]=\mathrm{E}\left[Y_{1 i}(t) Y_{2 j}(t)\right]=0, \quad i, j=1,2, \ldots, \quad i \neq j .
$$

And by the property of orthogonal functions:

$$
\mathrm{E}\left[Y_{1 i}(t) Y_{2 i}(t)\right]=0, \quad i=1,2, \ldots
$$

Substituting equations (14) and (15) into equation (13), one obtains

$$
\mathrm{E}\left[Y^{2}(t)\right]=\frac{a_{0}^{2}}{4}+\frac{1}{2} \sum_{i=1}^{\infty}\left(a_{i}^{2}+b_{i}^{2}\right) .
$$

$\mathrm{E}\left[Y^{2}(t)\right]$ is a finite number provided the corresponding deterministic Fourier series is convergent based on the well-known Parseval equality. 


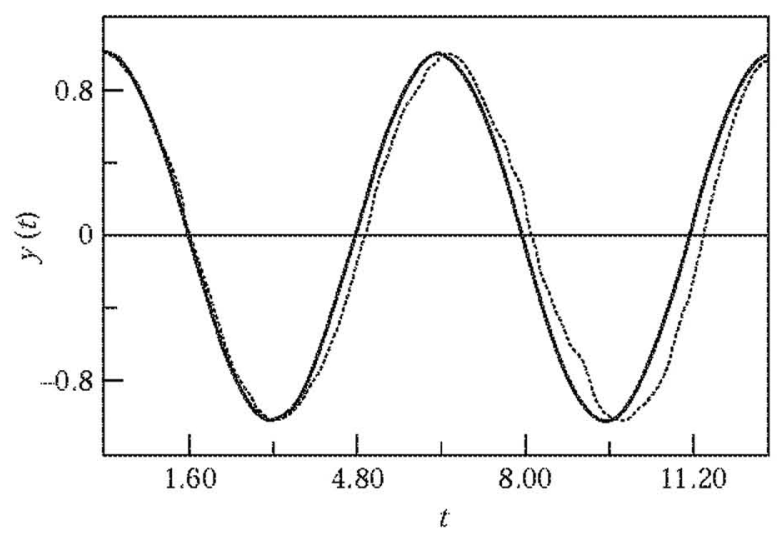

Figure 1. Simulation of harmonic wave with phase modulation: $D=0 \cdot 01 ;-$, , harmonic; $\cdots$, harmonic with noise.

Similarly, the auto-correlation function of $Y(t)$ can be found:

$$
R_{Y}(\tau)=\frac{a_{0}^{2}}{4}+\sum_{i=1}^{\infty} \frac{1}{2}\left(a_{i}^{2}+b_{i}^{2}\right) \cos \left(\mathrm{i} \omega_{0} \tau\right) \mathrm{e}^{\left(-D_{i} / 2\right)|\tau|}
$$

From the above results, one may conclude that the random Fourier series $Y(t)$ is generally a non-zero mean stationary random process, which is convergent in the mean square sense. For detailed derivation of equations (16) and (17), please see Appendix A.

\section{SIMULATION OF RANDOMLY DISORDERED PERIODIC PROCESSES}

Some graphic samples of the proposed model, $Y(t)$, are presented in Figures 1-6. These results are based on Monte Carlo simulation. Figure 1 shows the time history of a single harmonic excitation with small phase modulation. The phase shift caused by random phase modulation can be clearly observed.

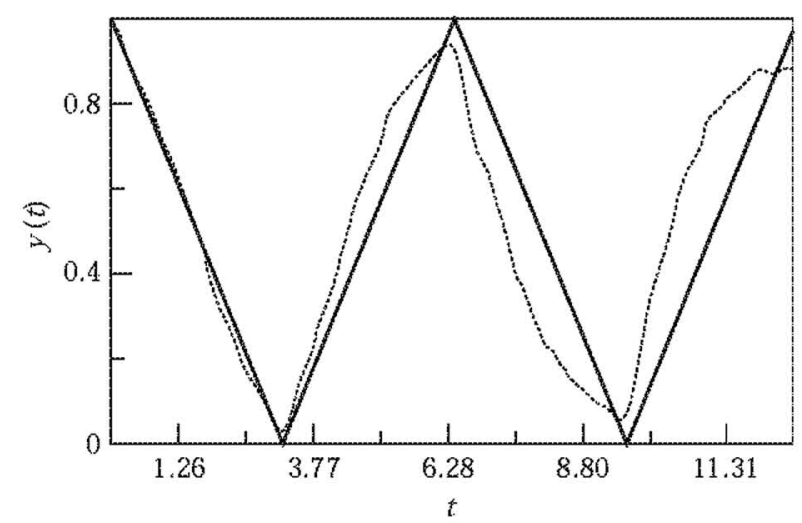

Figure 2. Simulation of random Fourier series for a triangular wave with small disturbance. For low noise $D(i)=0.01, i=1, \ldots, 6$. - Original signal $f_{t}(t) ; \cdots$, , Fourier series with noise, $Y_{t}(t)(N=6)$. 


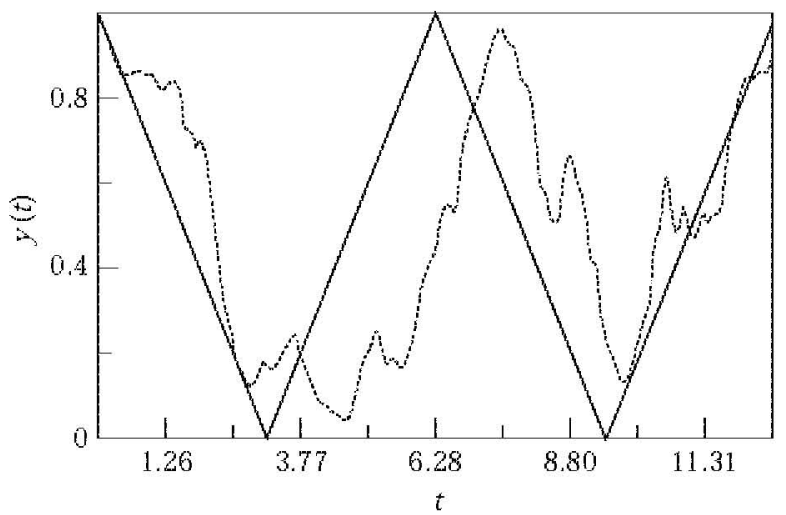

Figure 3. Simulation of random Fourier series for a triangular wave with medium disturbance. For medium noise $D(i)=0.25, I=1, \ldots, 6$. - Original signal $f_{t}(t), \cdots$, , Fourier series with noise, $Y_{t}(t)(N=6)$.

Figure 2 presents a sample for a disordered triangle waveform with a period of $2 \pi$. The Fourier expansion of the deterministic periodic counterpart is give by

$$
f_{i}(t)= \begin{cases}-i / \pi+1, & 0 \leqslant t<\pi \\ t / \pi-1, & \pi \leqslant t \leqslant 2 \pi\end{cases}
$$

The random Fourier series of the triangle waveform is

$$
Y_{t}(t)=\frac{1}{2}+\frac{4}{\pi^{2}} \sum_{i=1}^{\infty} \frac{\cos \theta_{i}(t)}{(2 i-1)^{2}}, \quad \frac{\mathrm{d} \theta_{i}(t)}{\mathrm{d} t}=2 i-1+\xi_{i}(t), \quad i=1,2, \ldots,
$$

where $\xi_{i}(t)$ is the random phase disturbance.

Samples of disordered triangle waveforms with three different levels of random disturbance are illustrated in Figures 2-4. Considering that $f_{i}(t)$ has $C^{0}$ continuity and the truncation error of $f_{t}(t)$ with six terms is negligible, six terms are also used in the simulation of $Y_{t}(t)$. While a sample with small random disturbance in Figure 2 or a sample with medium disturbance in Figure 3 may still be recognized as a disordered triangular waveform, the triangular pattern in the result for large phase modulation, as shown in Figure 4, disappears and the sample becomes more noise-like, as expected.

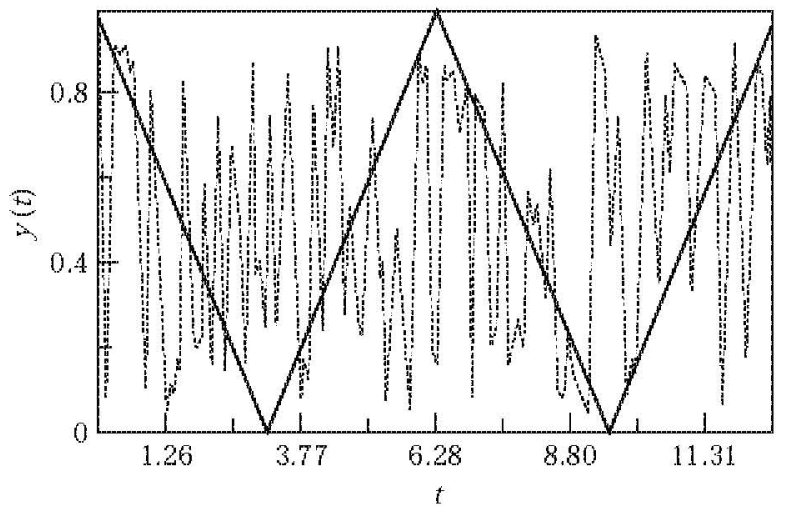

Figure 4. Simulation of random Fourier series for a triangular wave with large disturbance. For large noise $D(t)=25, i=1, \ldots, 6$. — , Original signal $f_{t}(t) ; \cdots$, Fourier series with noise, $Y_{t}(t)(N=6)$. 


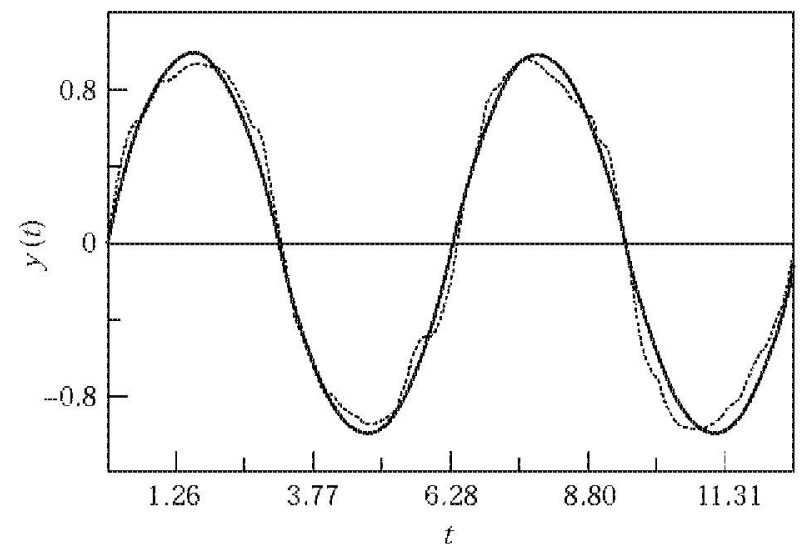

Figure 5. Simulation of random Fourier series for a quadratic wave with random disturbance. $D(i)=0 \cdot 01$, $i=1, \ldots, 5$. Original signal $f_{q}(t) ; \cdots$, , Fourier series with noise, $Y_{q}(t)(N=5)$.

Figure 5 gives an example of a quadratic wave with a period of $2 \pi$, which has $C^{1}$ continuity. A piecewise expression of the quadratic waveform is given by

$$
f_{q}(t)= \begin{cases}4 t(\pi-t) / \pi^{2}, & 0 \leqslant t<\pi \\ 4(t-\pi)(t-2 \pi) / \pi^{2}, & \pi \leqslant t \leqslant 2 \pi\end{cases}
$$

The function is expected to have a better convergence rate. Using only five terms in its Fourier series, the truncation error can hardly be seen. The small phase disturbance results in a phase shift from the original signal. The Fourier expansion of the quadratic wave with phase modulation is

$$
Y_{q}(t)=\frac{32}{\pi^{3}} \sum_{i=1}^{\infty} \frac{\sin \theta_{i}(t)}{(2 i-1)^{3}}, \quad \frac{\mathrm{d} \theta_{i}(t)}{\mathrm{d} t}=2 i-1+\xi_{i}(t), \quad i=1,2, \ldots
$$

Figure 6 depicts the case of a saw-tooth wave with a period of $2 \pi$ :

$$
f_{s}(t)=t / 2 \pi, \quad 0 \leqslant t \leqslant 2 \pi
$$

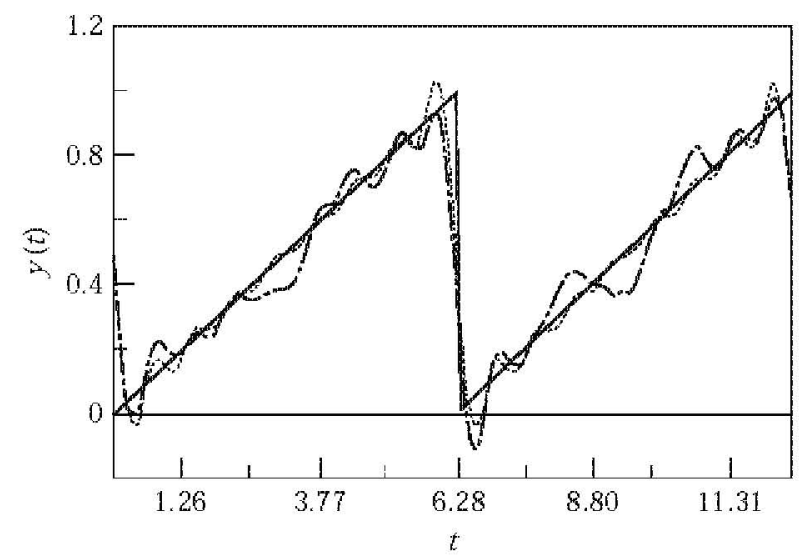

Figure 6. Simulation of random Fourier series for a sawtooth wave with random disturbance. $D(i)=0 \cdot 01$, $i=1, \ldots, 8$. - Original signal $f_{s}(t) ; \cdots$, , Fourier series $(N=8) ;-\cdots,-$, , Fourier series with noise $Y_{s}(t)$ $(N=8)$. 
The corresponding random Fourier series is expressed as

$$
Y_{s}(t)=\frac{1}{2}-\frac{1}{\pi} \sum_{i=1}^{\infty} \frac{\sin \theta_{i}(t)}{i}, \quad \frac{\mathrm{d} \theta_{i}(t)}{\mathrm{d} t}=i+\xi_{i}(t), \quad i=1,2, \ldots
$$

Eight terms are used in the simulation. Since the original waveform has discontinuity of the first kind at $t=2 m \pi$, where $m$ is an integer, the convergence of its Fourier series is much slower than in previous examples. The fluctuations from the original waveform are relatively large in comparison to the other cases of small disturbances. In order to distinguish sources of error caused by truncation and random disturbance, results for the corresponding deterministic Fourier series with the same number of terms is also presented for comparison.

\section{APPLICATION}

An application of general periodic excitation with uncertainty is demonstrated by the vibration analysis of a hydraulic control system in reference [11]. The valve and its elastic stem are modeled as a linear single-degree-of-freedom damped spring-mass system, as shown in Figure 7.

The fluid pressure on the valve changes with the amount of the opening or closing of the valve and is represented by a general periodic function with random phase modulation $Y(t)$. The differential equation describing the hydraulic control system subject to fluid pressure force is written as

$$
\begin{gathered}
\ddot{x}+2 \alpha \dot{x}+\Omega^{2} x=Y(t)=\frac{a_{0}}{2}+\sum_{i=1}^{\infty}\left(a_{i} \cos \theta_{i}+b_{i} \sin \theta_{i}\right), \\
\frac{\mathrm{d} \theta_{i}(t)}{\mathrm{d} t}=\mathrm{i} \omega_{0}+\xi_{i}(t),
\end{gathered}
$$

where $x(t)$ is the displacement response of the valve, $\Omega$ and $\alpha$ are, respectively, the natural frequency and damping constant of the system, and $Y(t)$ is assumed to be the force per unit mass without loss of generality. Equation (24) can be reformulated in the state space by introducing the following state variables:

$$
z_{1}=x, \quad z_{2}=\mathrm{d} x / \mathrm{d} t, \quad z_{2 i+1}=\cos \theta_{i}, \quad z_{2 i+2}=\sin \theta_{i}, \quad i=1, \ldots, n .
$$

where $n$ is the actual number of terms used in $Y(t)$. The corresponding stochastic Cauchy problem for the state variables $z_{i},(i=1, \ldots, 2 n+2)$ may be written as a set of the

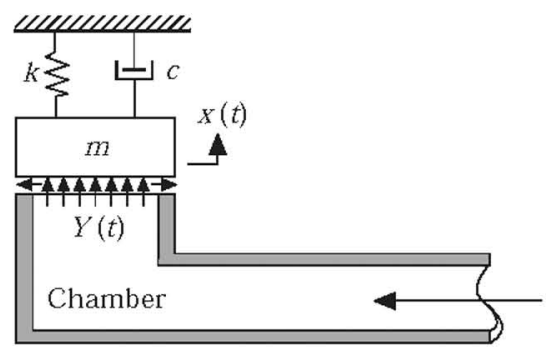

Figure 7. A schematic diagram for a hydraulic control system. 


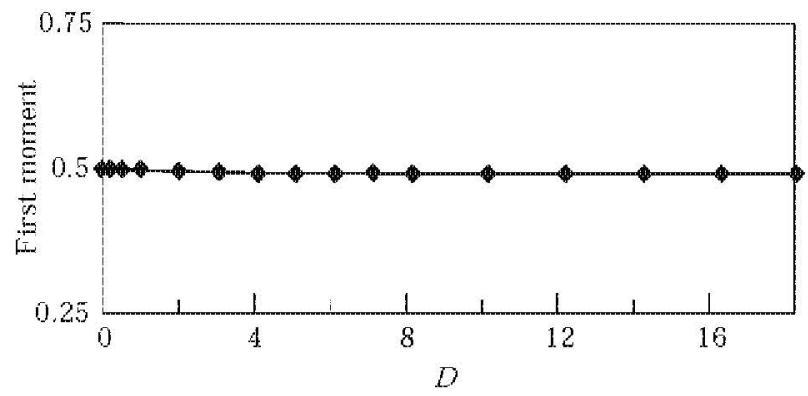

Figure 8. First order moment response of a hydraulic system subjected to a disturbed fluid pressure of triangular waveform: $\Omega=1, \alpha=0 \cdot 1, n=4$.

following $2(n+1)$ "physical" or Stratonovich stochastic differential equations:

$$
\begin{gathered}
\frac{\mathrm{d} z_{1}}{\mathrm{~d} t}=z_{2}, \quad \frac{\mathrm{d} z_{2}}{\mathrm{~d} t}=-\Omega^{2} z_{1}-2 \alpha z_{2}+\frac{a_{0}}{2}+\sum_{i=1}^{n}\left(a_{i z_{2 i+1}}+b_{i} z_{2 i+2}\right), \\
\frac{\mathrm{d} z_{2 i+1}}{\mathrm{~d} t}=-\left[\mathrm{i} \omega_{0}+\xi_{i}(t)\right] z_{2 i+2}, \quad \frac{\mathrm{d} z_{2 i+2}}{\mathrm{~d} t}=\left[\mathrm{i} \omega_{0}+\xi_{i}(t)\right] z_{2 i+1}, \quad i=1, \ldots, n .
\end{gathered}
$$

This SDE set is analyzed by the method of moments. Due to the autonomous representation of trigonometric functions in equation (26), this set is supplemented by obvious constraint equations:

$$
z_{2 i+1}^{2}+z_{2 i+2}^{2}=1, \quad i=1, \ldots, n .
$$

By appropriately applying the Ito differential rule and the mathematical expectation operator, a set of deterministic equations for various orders of the response moments can be derived. A computer algorithm has been written to generate all the coefficients in the moment equations, deal with constraint equations, and finally solve for different orders of moments of the response.

In the following numerical example, the pressure force $Y(t)$ has the triangle wave form defined by equation (21). $Y(t)$ is generally a non-zero mean excitation, therefore, the response moments of odd order are not necessarily zero. The stationary moment (including

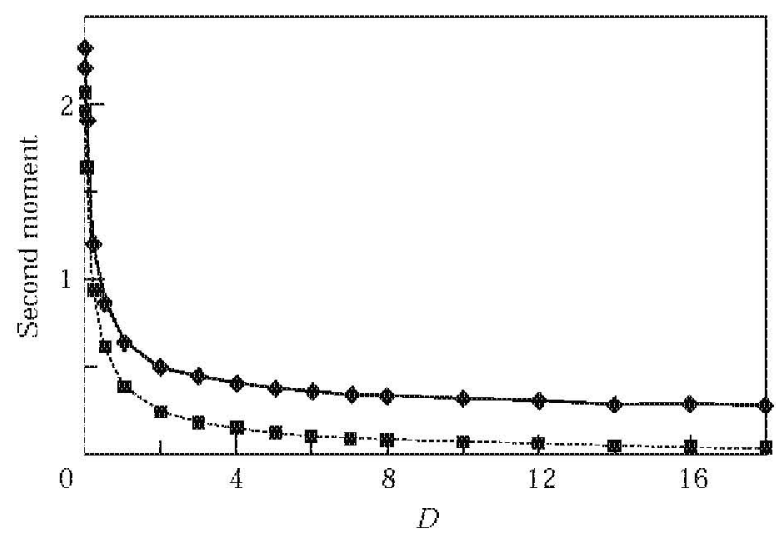

Figure 9. Second order moment response of a hydraulic system subjected to a disturbed fluid pressure of triangle waveform: $\Omega=1, \alpha=0 \cdot 1, n=4$. - Second moment; $\cdots$, , second central moment. 


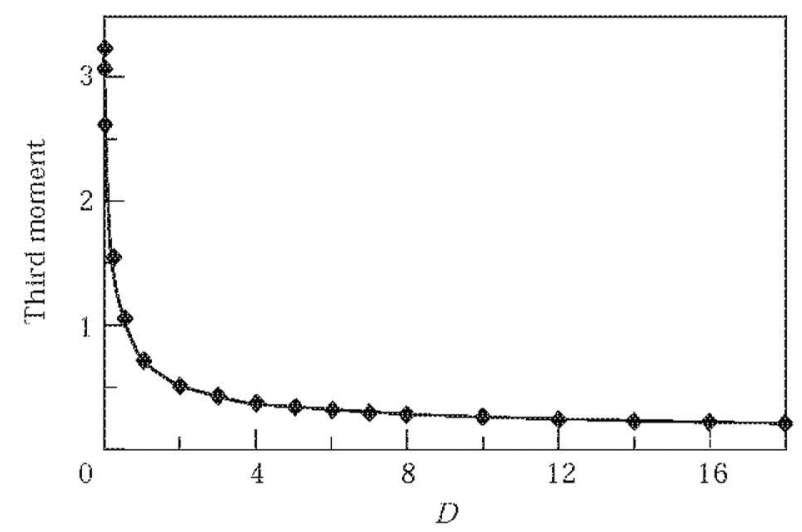

Figure 10. Third order moment response of a hydraulic system subjected to a disturbed fluid pressure of triangle waveform: $\Omega=1, \alpha=0 \cdot 1, n=4$.

central moment) responses of up to the fourth order are plotted versus phase modulation intensity $D$ in Figures 8-11. An identical phase modulation intensity in all harmonic terms is assumed in the present study. Numerical results show that the moment responses of valve are significantly reduced for $0<D<4$, which implies that the opening of the valve may become much smaller due to the random noise in the pressure force, even if it is very small. For large phase modulation, the moment responses change slowly. Let $m_{x}$ denote the mean value of $\mathrm{E}[x]$ : our calculation shows that $\mathrm{E}\left[\left(x-m_{x}\right)^{3}\right]$ is always zero. It implies that the skewness of the response process is zero, or the response is symmetric to its mean value in a probabilistic sense.

As mentioned before, the random process $Y(t)$ is generally non-Gaussian, so is the response of linear system. An excess factor is defined here to represent the non-Gaussian nature of the response:

$$
\left.\left.K=3-\mathrm{E}\left[\left(x-m_{x}\right)^{4}\right] /\left\{\mathrm{E}\left[x-m_{x}\right)^{2}\right)\right]\right\}^{2} .
$$

For a Gaussian process, $K=0$; for a deterministic harmonic function, $K=1 \cdot 5$. From Figure 12, one can see that the excess factor approaches zero as $D$ increases. This is the well-known normalization effect.

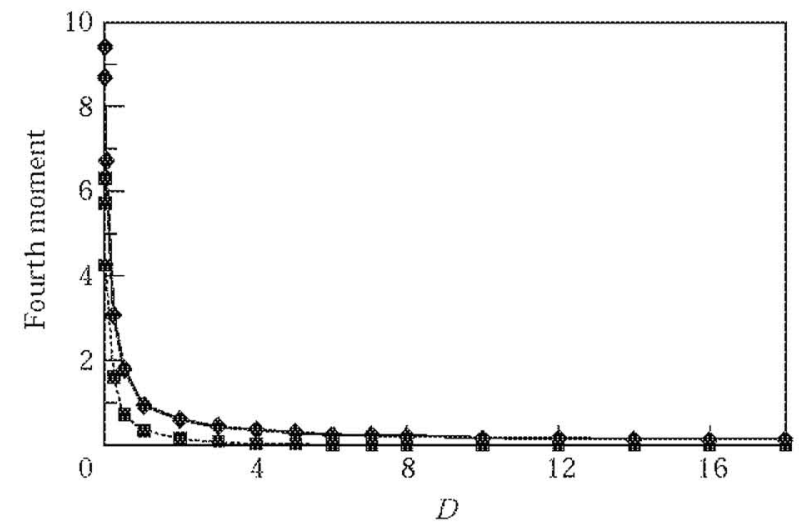

Figure 11. Fourth order moment response of a hydraulic system subjected to a disturbed fluid pressure of triangle waveform: $\Omega=1, \alpha=0 \cdot 1, n=4$. - Fourth moment; $\cdots$, fourth central moment. 


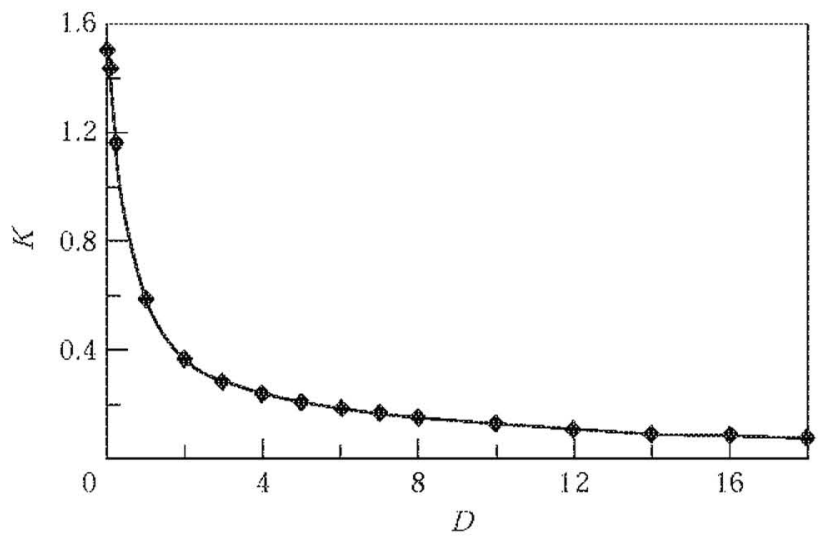

Figure 12. Excess factor of the random response of a hydraulic system subjected to a disturbed fluid pressure of triangle waveform: $\Omega=1, \alpha=0 \cdot 1, n=4$.

Numerical results plotted in Figures 13 and 14 show the effect of phase modulation intensity $D$ on the convergence rate of the second and fourth order moment response. A relative error is used as the measure of the convergence rate, which is defined as

Error $=[$ moment value $(n=5)-$ moment value $(n<5)] /$ moment value $(n=5)$,

where $n$ is the number of items included in the random Fourier series. The smaller is the error value, the faster is the response convergence. The moment value with $n=5$ is treated as the converged result in this case based on numerical calculation. Therefore, the error should be zero for $n \geqslant 5$.

Three different $D$ levels are used in the investigation of convergence rate, namely, $D=0$, $D=2$ and $D=10$. The defined errors of response moments of both second and fourth orders decrease with an increasing total number of terms in the random Fourier series and increase with increasing phase modulation intensity $D$. That is, the random disturbance in general periodic excitation results in slower convergence of the response moments.

The spectral density of valve response $S_{x}(\omega)$ is shown in Figure 15, which is calculated based on the excitation spectrum $S_{Y}(\omega)$ and the system frequency response function $H(\omega)$

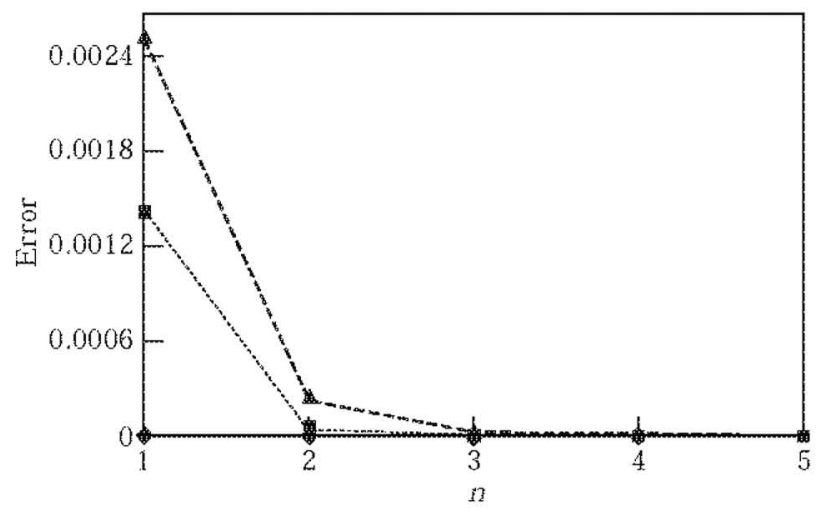

Figure 13. Convergence of the second order moment response for disturbed triangle waveform excitation: $\Omega=1, \alpha=0 \cdot 1 ;-, D=0 ; \cdots, D=2 ; \cdots, D=10$. 


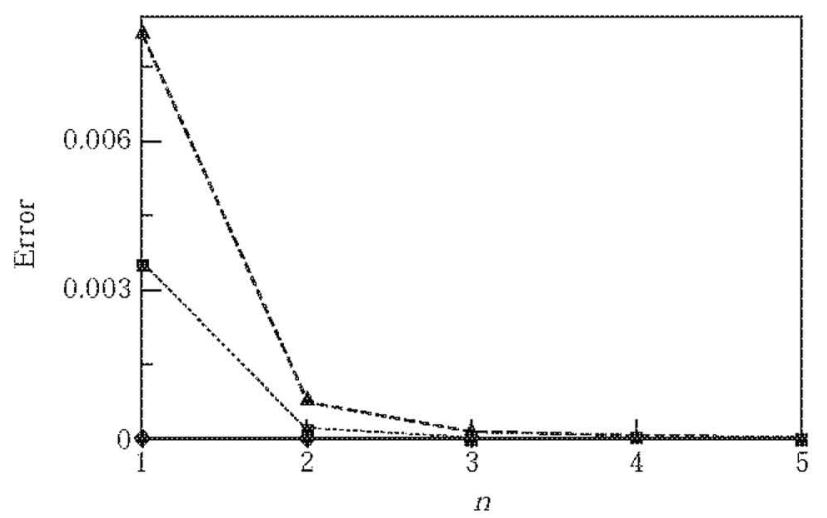

Figure 14. Convergence of fourth order moment response for disturbed triangle waveform excitation: $\Omega=1$, $\alpha=0 \cdot 1 ;-, D=0 ; \cdots, D=2 ; \cdots, D=10$.

by the following formula:

$$
S_{x}(\omega)=|H(\omega)|^{2} S_{Y}(\omega)
$$

where

$$
\begin{gathered}
|H(\omega)|^{2}=1 /\left[\left(\Omega^{2}-\omega^{2}\right)^{2}+4 \alpha^{2} \omega^{2}\right], \\
S_{Y}(\omega)=\sum_{i=1}^{n} S_{y i}(\omega)=\frac{a_{0}^{2}}{4} \delta(\omega)+\sum_{i=1}^{n} \frac{\left(a_{i}^{2}+b_{i}^{2}\right) D_{i}\left[\left(i \omega_{0}\right)^{2}+\omega^{2}+D_{i}^{2} / 4\right]}{4 \pi\left[\left\{\left(i \omega_{0}\right)^{2}-\omega^{2}+D_{i}^{2} / 4\right\}^{2}+\omega^{2} D_{i}^{2}\right]} .
\end{gathered}
$$

Three peaks are observed in the response spectrum curve, corresponding to the fundamental harmonic frequency of the excitation at $\omega=1$, system natural frequency at $\omega=2 \cdot 2$ and the second harmonic frequency at $\omega=3$. The third peak is almost invisible. Other higher order harmonics of the excitation are filtered by the system. It should be noted that there is a delta impulse with intensity $a_{0}^{2} / 4 \Omega^{4}$ at $\omega=0$ due to absence of random disturbance in the constant component or zeroth harmonic in the Fourier expansion of the excitation. For larger $D$ value, the response spectrum curve is flatter because the excitation spectrum becomes wider banded.

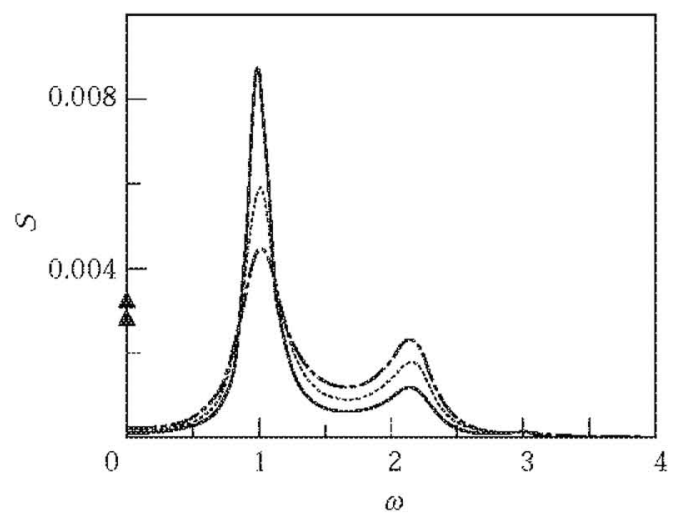

Figure 15. Spectral density of the response for disturbed trian gle waveform excitation: $\Omega=2 \cdot 2, \alpha=0 \cdot 22, n=4$. $\longrightarrow, D=0 \cdot 2 ; \cdots, D=0 \cdot 3 ;-\cdot-\cdot-\cdot-, D=0 \cdot 4$. Note that there is an impulse at $\omega=0$, i.e., $\left(a_{0}\right)^{2} \delta(\omega) / 4 \Omega^{4}$. 


\section{SUMMARY AND CONCLUSIONS}

A model of general periodic excitation with random disturbance is presented in this paper. The excitation model is represented by a random Fourier series with random disturbance in each term. Monte Carlo simulations of different types of waveform with random disturbance are illustrated. The nature of the proposed excitation process is discussed and the application of the model by random vibration analysis of a hydraulic valve system under a disordered periodic fluid pressure. Numerical results of moment responses of up to the fourth order, excess factor, and spectral density are presented.

Conclusions from the investigation are summarized as:

1. The random Fourier series presented in this paper, which models general periodic excitation with random disturbance, is in general a stationary, random process with a non-zero mean. It is mean-square convergent provided the corresponding deterministic Fourier series is convergent.

2. The existence of random disturbance can significantly reduce the moment responses of linear systems, as demonstrated for a hydraulic control system. The moment responses of the valve in the system are extremely sensitive to a small level of random disturbance.

3. Existence of the random disturbance reduces the convergence rate of the system moment response. It implies that more terms should be used in a random Fourier series to approximate general disordered periodic excitation.

4. The random disturbance in the present model affects the Gaussian nature of the system response. The larger is the disturbance, the closer is the system response process to the corresponding Gaussian process in the sense of up to the fourth moment.

The model may be further developed to include random disturbance in amplitudes of harmonics and correlaton between disturbances in different harmonics. The model and numerical procedure presented in this paper also apply to linear multiple-degree-of-freedom (MDOF) systems. The computational effort for fourth order MDOF system response moment will increase drastically since the constrain equation (27) and cross-correlations among excitations need to be considered. The computation effort may be significantly reduced by the approach of modal analysis, which uncouples the original MDOF system into a set of SDOF systems if the response of a few modes is dominant. The work can be readily extended to random disturbances of the shot-noise type. An application of the model to non-linear systems and further development to non-periodic processes is under investigation.

\section{ACKNOWLEDGMENT}

This research is sponsored partly by ONR-URI Grant No. N00014-93-1-0917. This support is gratefully acknowledged.

\section{REFERENCES}

1. Z. K. Hou, Y. S. Zhou, M. F. Dimentberg and M. NoORI 1995 Accepted by Probabilistic Engineering Mechanics. A stationary model for periodic excitation with uncorrelated random disturbances.

2. Z. K. Hou, Y. S. ZHou, M. F. Dimentberg and M. Noori 1996 ASCE Journal of Engineering Mechanics (to appear). Random response to periodic excitation with correlated disturbances. 
3. M. F. DimentbeRg 1988 Statistical Dynamics of Nonlinear and Time-Varying System, England: Research Studies Press.

4. W. WEDIG 1989 Nonlinear Dynamics in Engineering Systems (Editor W. Schiehlen). Berlin: Springer Verlag; pp. 337-344. Analysis and simulation of nonlinear stochastic systems.

5. M. F. DimentberG 1991 In Proceedings of the 5th International Conference on Flow-Induced Vibrations, Brighton, UK, 207-209. A stochastic model of parametric excitation of a straight pipe due to slug flow of a two-phase fluid.

6. M. F. Dimentberg 1992 Probabilistic Engineering Mechanics 7, 131-134. Stability and subcritical dynamics of structures with spatially disordered traveling parametric excitation.

7. Y. K. LIN and Q. C. LI 1993 Journal of Engineering Mechanics 119, 113-127. New stochastic theory for bridge stability in turbulent flow.

8. M. F. Dimentberg, Z. K. Hou, M. N. NoORI and W. Zhang 1993 ASME special volume Recent Development in the Mechanics of Contima. Non-Gaussian response of a single-degree-offreedom system to a periodic excitation with random phase modulation.

9. Z. K. Hou, Y. S. ZHOU, M. F. DIMENTBERG and M. NOORI 1995 Probabilistic Engineering Mechanics 10, 73-81. A non-stationary stochastic model for periodic excitation with random phase modulation.

10. R. V. ChURCHILL and J. W. BRown 1978 Fourier Series and Boundary Value Problems. New York: McGraw-Hill; third edition.

11. S. S. RAO 1995 Mechanical Vibrations. Addison-Wesley Publishing Company Inc; third edition.

\section{APPENDIX: DERIVATION OF EQUATIONS (16) AND (17)}

The following derivation is based on the assumption that disturbances in different harmonic terms are independent of each other. Let

$$
Y_{1 i}(t)=\cos \theta_{i}(t), \quad Y_{2 i}(t)=\sin \theta_{i}(t), \quad i=1,2, \ldots
$$

Assume $\theta_{i}(t)$ is a stationary random process uniformly-distributed in $[0,2 \pi]$. The mean value of $Y_{1 i}(t)$ thus is

$$
\mathrm{E}\left[\boldsymbol{Y}_{1 i}\right]=\int_{0}^{2 \pi} \frac{1}{2 \pi} \cos \theta_{i} \mathrm{~d} \theta=0
$$

Similarly,

$$
\mathrm{E}\left[Y_{2 i}\right]=0 .
$$

The mean square value of $Y_{1 i}(t)$ is

$$
\mathrm{E}\left[Y_{1 i}^{2}\right]=\int_{0}^{2 \pi} \frac{1}{2 \pi} \cos ^{2} \theta_{i} \mathrm{~d} \theta=\frac{1}{2}
$$

A similar result is obtained for $Y_{1 i}(t)$, i.e.,

$$
\mathrm{E}\left[Y_{2 i}^{2}\right]=1 / 2 .
$$

The mean value of $Y_{1 i}(t) \cdot Y_{2 i}(t)$ is

$$
\mathrm{E}\left[Y_{1 i} Y_{2 i}\right]=\int_{0}^{2 \pi} \frac{1}{2 \pi} \sin \theta_{i} \cos \theta_{i} \mathrm{~d} \theta=\frac{1}{4 \pi} \int_{0}^{2 \pi} \sin 2 \theta_{i} \mathrm{~d} \theta=0 .
$$

The assumption that random disturbance $\xi_{i}(t)$ in each harmonic term are independent of each other leads to

$$
\mathrm{E}\left[Y_{1 i} \boldsymbol{Y}_{1 j}\right]=\mathrm{E}\left[\boldsymbol{Y}_{2 i} \boldsymbol{Y}_{2 j}\right]=\mathrm{E}\left[Y_{1 i} \boldsymbol{Y}_{2 j}\right]=0, \quad i, j=1,2, \ldots, \quad i \neq j
$$


The mean square value of $Y(t)$ is

$$
\begin{aligned}
\mathrm{E}\left[Y^{2}\right]= & \mathrm{E}\left\{\frac{a_{0}}{2}+\sum_{i=1}^{\infty}\left[a_{i} Y_{1 i}+b_{i} Y_{2 i}\right]\right\}^{2} \\
= & \frac{a_{0}^{2}}{4}+a_{0} a_{i} \sum_{i=1}^{\infty} \mathrm{E}\left[Y_{1 i}\right] \\
& +a_{0} b_{i} \sum_{i=1}^{\infty} \mathrm{E}\left[Y_{2 i}\right]+\left\{\sum_{i=1}^{\infty}\left[a_{i} Y_{1 i}+b_{i} Y_{2 i}\right]\right\}^{2} .
\end{aligned}
$$

By equations (A2) and (A3), the second and third terms in equation (A8) are zero and the last term becomes

$$
\begin{aligned}
\mathrm{E}\left\{\sum_{i=1}^{\infty}\left[a_{i} Y_{1 i}+b_{i} Y_{2 i}\right]\right\}^{2}= & \sum_{i=1}^{\infty} a_{i}^{2} \mathrm{E}\left[Y_{1 i}^{2}\right]+\sum_{i=1}^{\infty} b_{i}^{2} \mathrm{E}\left[Y_{2 i}^{2}\right]+2 \sum_{i=1}^{\infty} a_{i} b_{i} Y_{1 i} Y_{2 i}+2 \sum_{i=1, j=1, i \neq j}^{\infty} \\
& \times\left(a_{i} a_{j} Y_{1 i} Y_{1 j}+a_{i} b_{j} Y_{1 i} Y_{2 j}+b_{i} a_{j} Y_{2 i} Y_{1 j}+b_{i} b_{j} Y_{2 i} Y_{2 j}\right) .
\end{aligned}
$$

Based on equations (A4)-(A7), equation (A9) can be further simplified as

$$
\mathrm{E}\left\{\sum_{i=1}^{\infty}\left[a_{i} \boldsymbol{Y}_{1 i}+b_{i} \boldsymbol{Y}_{2 i}\right]\right\}^{2}=\frac{1}{2} \sum_{i=1}^{\infty}\left(a_{i}^{2}+b_{i}^{2}\right) .
$$

Finally, substitute equation (A10) into equation (A8):

$$
\mathrm{E}\left[Y^{2}(t)\right]=\frac{a_{0}^{2}}{4}+\frac{1}{2} \sum_{i=1}^{\infty}\left(a_{i}^{2}+b_{i}^{2}\right) .
$$

For the auto-correlation function of $Y(t)$, the derivation process is similar to that of mean square. By eliminating cross term expectations, one obtains

$$
\begin{aligned}
R_{Y}(\tau)= & \mathrm{E}[Y(t) Y(t+\tau)] \\
= & \mathrm{E}\left\{\frac{a_{0}^{2}}{4}+\sum_{i=1}^{\infty} a_{i}^{2} \cos \theta_{i}(t) \cos \theta_{i}(t+\tau)\right. \\
& \left.+\sum_{i=1}^{\infty} b_{i}^{2} \sin \theta_{i}(t) \sin \theta_{i}(t+\tau)\right\} \\
= & \frac{a_{0}^{2}}{4}+\sum_{i=1}^{\infty}\left[a_{i}^{2} R_{Y 1 i}(\tau)+b_{i}^{2} R_{Y 2 i}(\tau)\right] \\
= & \frac{a_{0}^{2}}{4}+\frac{1}{2} \sum_{i=1}^{\infty}\left(a_{i}^{2}+b_{i}^{2}\right) \cos (\mathrm{i} \omega \tau) \mathrm{e}^{\left(-D_{i} / 2\right)|\tau|} .
\end{aligned}
$$

